\title{
Simon Meier*
}

\section{Wutreden - Konstruktion einer Gattung in den digitalen Medien}

DOI 10.1515/zgl-2016-0002

\begin{abstract}
This paper proposes a reception-oriented and discursive concept of genre by using the example of „Wutreden“, i. e. public and medially distributed rants and their coverage in (online) media and Web 2.0. On the basis of a corpus of video recordings and transcriptions of Wutreden as well as comments made by journalists and users, it is shown that neither their linguistic features nor their situational functions qualify Wutreden as a distinct genre. Rather, the medial and internetbased recontextualisations of Wutreden build an orienting framework for the reception of discourse. It is these recontextualisations that put disparate speech events in order, so that they can be received as instances of one single genre. The analysis of accompanying discourse like media reports, user comments, and tweets as well as the multimodal surroundings of the respective videos shows, that such documents of reception reflect a genre awareness of Wutreden which will shape their reception as well as the follow-up discourse. The increase of Wutreden in the recent past as appears to be as a result of altered conditions of reception in online environments - conditions, which the linguistic study of genre and genre change within digital media should not neglect.
\end{abstract}

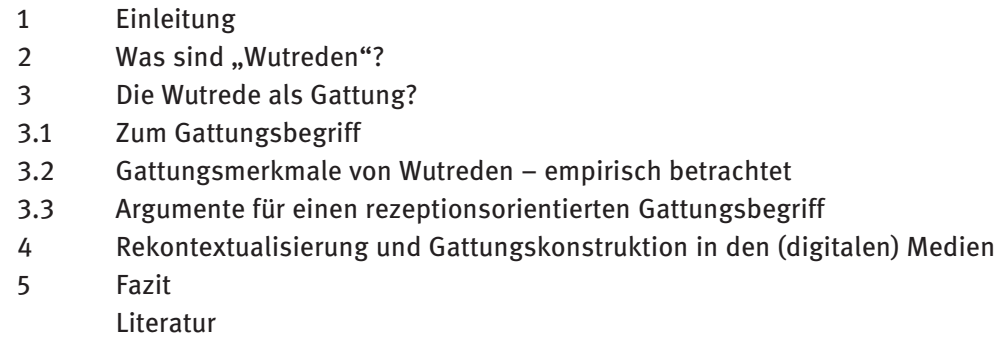

\footnotetext{
*Kontaktperson: Dr. Simon Meier: Technische Universität Berlin, Institut für Sprache und Kommunikation, Fachgebiet Allgemeine Linguistik, Straße des 17. Juni 135, D-10623 Berlin, E-Mail: simon.meier@tu-berlin.de
} 


\section{Einleitung}

Neuere Arbeiten zur Textsortenlinguistik oder - in einem erweiterten Sinne - zur linguistischen Kommunikationstypologie (vgl. Habscheid 2011b) nehmen zunehmend diachrone Aspekte in den Blick (vgl. etwa Hauser et al. 2014). Auch unabhängig von dezidiert sprachgeschichtlichen Fragestellungen wird dafür plädiert, Textsorten weniger als objektive und als sprachliche Merkmalskonfigurationen eindeutig beschreibbare Gebilde denn als „von einer Kommunikationsgemeinschaft diskursiv hervorgebrachte und tradierte Größen“ (Luginbühl/Perrin 2011: 577) aufzufassen, und zwar nicht nur hinsichtlich ihrer Emergenz, sondern auch hinsichtlich ihrer Kategorisierung und Terminologisierung (vgl. Holly 2002: 2452). Linguistische Textsortentypologien seien deshalb an die ihrerseits historisch und kulturell situierte Typisierungspraxis der Kommunizierenden selbst rückzubinden (vgl. Habscheid 2011a: 16f.). Die diachrone Perspektive ist hier bereits in den Textsortenbegriff selbst eingelassen. Die letzthin oft gestellte Frage nach der Kulturalität von Textsorten (vgl. Fix 2008: 28; Luginbühl 2014b: 40-43) als den sprachlichen Erscheinungsformen kollektiver Handlungs- und Situationstypen öffnet ebenfalls den Blick für diachrone Aspekte. Indem Textsorten als „sozialhistorisch entstandene und tradierte, damit auch kulturspezifisch geprägte [...] Formen sprachlich-kommunikativen Handelns“ (Krause 2000: 48) aufgefasst werden, die „kondensierte Reflexe kommunikativer Aufgaben“ (Heinemann 2000: 515) darstellen, ist die Frage nach den Gründen für die Entstehung und den Wandel bestimmter Textsorten wenigstens implizit mitgestellt.

Im Hinblick auf technologische Innovationen und veränderte mediale Kontexte sind nun in der Tat vielfältige Wandelprozesse zu konstatieren, die bei einer adäquaten Beschreibung gegenwärtiger textueller Phänomene wie multimodal angereicherten und hypertextuell verknüpften Texten oder auch Hybridisierungstendenzen von Gattungen berücksichtigt werden müssen (vgl. Heyd 2009; Fix 2014; Tienken 2015). Gerade zur Erfassung dieser Wandelprozesse aufgrund von veränderten technisch-medialen Kommunikationskonstellationen wird in vielen Ansätzen (vgl. etwa Dürscheid 2005; Imo 2011) die Theorie der kommunikativen Gattungen (vgl. etwa Günthner/Knoblauch 1994; Günthner 1995) herangezogen, die auch für das umrissene Verständnis von (mündlichen wie schriftlichen) Textsorten als routinisierten Lösungen wiederkehrender Aufgaben wichtige Impulse gegeben hat. 
Im Folgenden soll mit der sogenannten Wutrede eine mündliche ,Textsorte‘ bzw. ,Gattung ${ }^{61}$ in den Blick genommen werden, die sich einerseits in eben diese Untersuchungsrichtung einfügt, da sie, wie ich zeigen werde, ihre charakteristische Konjunktur im Kontext der digitalen Medien erfahren hat. ${ }^{2}$ Andererseits ist die Sachlage hinsichtlich der Wutrede nochmals anders nuanciert, da diese mitnichten (wie etwa die Gattung Expertenchat, vgl. Dürscheid 2005) einen tatsächlich neuen Typ sprachlich-kommunikativen Handelns darstellt. Sie trifft aber auf veränderte Rezeptionsbedingungen und ist mithin in veränderte und an einer Vielzahl von Rezeptionszeugnissen ablesbare interpretative Verfahren eingebettet, die die Wutrede als Gattung erst konstituieren - und zwar weniger für die wissenschaftlich Analysierenden, die an der Gattungsidentität der Wutrede begründete Zweifel haben mögen, als vielmehr für die Diskursteilnehmer selbst. Diesen gattungskonstruktiven Praktiken gilt das Interesse der folgenden Ausführungen.

Der Aufsatz verfolgt somit drei aufeinander bezogene Ziele. Erstens soll das (noch zu beschreibende) Phänomen der erstaunlichen Konjunktur von Wutreden in jüngerer Zeit aus sprachwissenschaftlicher Sicht beschrieben und erklärt werden. Damit soll zweitens exemplarisch ein medialer Kommunikationstyp erörtert werden, der in bisherigen Forschungsarbeiten zu neuen bzw. veränderten Textsorten und Gattungen im Kontext digitaler Medien eher vernachlässigt wird (vgl. aber Androutsopoulos 2015): Lag der Fokus bisher eher auf genuin internetbasierten Textprodukten und kommunikativen Praktiken, die schon in formaler und funktionaler Hinsicht wenigstens verändert oder gar neu sind (Blogs, Chats, Internetforen, Newsgroups etc.; vgl. hierzu etwa Giltrow/Stein 2009a), handelt es sich hier um eine Gattung, die sich durch mediale, insbesondere internetbasierte Rekontextualisierungen konstituiert. Dies liefert drittens empirische wie theoretische Argumente für eine stärker rezeptions- und diskursorientierte Fassung des Gattungsbegriffs, wie sie (unter ganz anderen Vorzeichen) etwa von Vertretern

\footnotetext{
1 Eine strikte Unterscheidung von Textsorten und Gattungen wird hier nicht vorgenommen (vgl. hierzu etwa Dammann 2000: 547). Wenn aber im Folgenden eher von Gattungen als von Textsorten die Rede ist, dann geschieht das vornehmlich aus Gründen der Anschlussfähigkeit an den unten (Kap. 3.3) aufgegriffenen Genrebegriff der amerikanischen Linguistic Anthropology. Zudem suggeriert der Terminus „Textsorte“ trotz jüngerer Entwicklungen (vgl. zusammenfassend Hauser/Luginbühl 2015: 13f.) eben immer noch jene Beschränkung auf schriftliche und formal-funktional exhaustiv zu bestimmende Typen, die hier gerade vermieden werden soll. Alles, was hier entwickelt wird, beansprucht aber auch textsortenlinguistische Relevanz.

2 „Digitale Medien“ dient hier als Oberbegriff für sämtliche Formen internetbasierter Medienangebote und umfasst also Onlinezeitungen (vgl. Burger/Luginbühl 2014: 451-469) ebenso wie das Web 2.0 (vgl. Marx/Weidacher 2014: 66-71). Durch die standardmäßigen Kommentarfunktionen und die Nutzung der sozialen Netzwerke auch durch professionelle Redaktionen vermischen sich diese beiden Bereiche zusehends.
} 
der rezeptionsästhetischen Gattungstheorie (vgl. Jauß 1977), vor allem aber der Linguistic Anthropology (vgl. etwa Briggs/Bauman 1992) vorgeschlagen wurde. Für das in der kulturwissenschaftlich orientierten Textlinguistik seit längerem zu beobachtende Interesse an den Interpretationsleistungen der Rezipierenden bei der Emergenz von Textsorten (vgl. Luginbühl 2014a: 308) kann gerade ein so konturierter Gattungsbegriff fruchtbar gemacht werden.

Im Folgenden werde ich zunächst darlegen, was im aktuellen Sprachgebrauch unter „Wutreden“ verstanden wird. Anschließend werde ich diskutieren, inwiefern die Wutrede als (kommunikative) Gattung beschrieben werden kann. Auf der Grundlage von transkribierten Aufzeichnungen von Wutreden wie auch von Presseberichten über Wutreden werde ich ihre typischen Gattungsmerkmale aufzeigen. Aufgrund der sich hierbei ergebenden Probleme werde ich einen rezeptions- und diskursorientierten Gattungsbegriff entwickeln, der das gattungskonstruktive Potenzial von sog. Rekontextualisierungen betont, welche im Kontext v. a. der digitalen Medien besondere Ausformungen annehmen. Abschließend werde ich einige Konsequenzen eines so konturierten Gattungsbegriffs für das Vorhaben einer diachronen Textsortenlinguistik diskutieren.

\section{Was sind „Wutreden“?}

Im aktuellen öffentlichen Sprachgebrauch bezeichnet der Ausdruck Wutrede für gewöhnlich öffentliche, aber spontan und emotional-impulsiv gehaltene Reden von Funktionsträgern wie Fußballtrainern oder, insbesondere in jüngerer Zeit, auch Politikern, die in ihren Reden meist sehr deutliche Kritik formulieren. Der Ausdruck ist noch recht jung und geht auf die Berichterstattung zu Giovanni Trapattonis berühmter Pressekonferenz vom 10. März 1998 zurück.

(1) „Schwach wie eine Flasche leer!“ Wie ein Peitschenhieb geißelte diese Aussage bei der Wut-Rede von Giovanni Trapattoni die erschreckend schwachen Vorstellungen der Mannschaft. (BILD, 14.3.1998) ${ }^{3}$

Wie sich verschiedenen Pressedatenbanken und Korpora (Factiva, Lexis, DeReKo dwds.de) entnehmen lässt, wird der Ausdruck aber zunächst nur selten gebraucht, bis sich am 6. September 2003 der damalige Fußballbundestrainer Rudi

3 Das ist nach meinen Recherchen der früheste Beleg. Das Grimm'sche Deutsche Wörterbuch (Bd. 30, Sp. 2488) enthält zwar auch schon einen Eintrag mit dem auf das Jahr 1818 datierten Beleg „Passow hat wider Menzels Wutrede einige Worte drucken lassen“ (Meyer 1913: 106), doch erst nach 1998 findet das Lexem in der heute relevanten Bedeutung Eingang in den Wortschatz. 
Völler im spielanschließenden Interview gegen die Kritik der TV-Experten Netzer und Delling zur Wehr setzt und damit ein großes Medienecho verursacht. Nun wird der Ausdruck erstmals von einer Vielzahl von Zeitungen verwendet, und sein Gebrauch nimmt über die Jahre stetig zu. Ungefähr ab 2008 vergeht kaum eine Woche ohne Bericht über eine neue Wutrede. Seit 2009 wird das Wort Wutrede im Duden geführt.

Eine Aufschlüsselung nach Themen der über 3000 Presseartikel, welche die Datenbank Factiva für den Suchbegriff „Wutrede“ ausgibt, zeigt, dass mit ca. 1500 Treffern rund die Hälfte aller Wutreden - also Reden, die in der öffentlichen Rezeption als Wutreden bezeichnet werden - im fußballerischen Kontext gehalten wurden und heute noch werden. Nach Trapattoni lieferten $u$. a. die Trainer Thomas Doll und Bruno Labbadia vielzitierte Wutreden. Eine Prüfung der Einzelbelege zeigt aber, dass ungefähr ab 2005 immer wieder auch Politikerreden (Bsp. 2) oder auch der Auftritt des Literaturkritikers Marcel Reich-Ranicki beim Deutschen Fernsehpreis (Bsp. 3) als Wutreden bezeichnet werden:

(2) Zuletzt hatte sich der Ostdeutsche bei seiner Fraktion mit einer Wutrede zur Vertrauensfrage von Bundeskanzler Gerhard Schröder unbeliebt gemacht. (Frankfurter Rundschau, 11.7.2005)

(3) Mit seiner neunminütigen, wuchtigen Wutrede gegen den „Blödsinn“ im Unterhaltungsfernsehen bei der Gala zum Deutschen Fernsehpreis hat sich Marcel Reich-Ranicki zum Ombudsmann des leidenden Fernsehvolks gemacht. (Hannoversche Allgemeine, 14.10.2008)

Jüngere und vielbeachtete Beispiele für Wutreden von Politikern sind einmal die Reaktion von Außenminister Frank-Walter Steinmeier auf Zwischenrufe während einer Kundgebung im Mai 2014 und einmal die Reaktion des Politikers Christian Lindner auf einen Zwischenruf während seiner Rede vor dem Landtagsparlament im Februar 2015. Beide Reden bzw. Redeteile wurden in der Folge zunächst über die sozialen Netzwerke und später auch durch die etablierten (Online-)Medien ausdrücklich als „Wutreden“ verbreitet und ausführlich diskutiert. Überhaupt hat sich in jüngerer Zeit der öffentliche Diskurs über Wutreden ins Internet verlagert. Dies betrifft einerseits die professionelle Berichterstattung über Onlinemedien, wo die Videoaufzeichnungen der Wutreden häufig gleich mitpubliziert werden, ${ }^{4}$ andererseits die Kommentierungs- und Weiterverbreitungsaktivitäten der Mediennutzenden über die interaktiven Funktionen des Web 2.0. „Das Netz liebt

4 Gerade über die erwähnten Wutreden der Politiker Steinmeier und Lindner wurde in den Onlinemedien mit den eingebetteten, in beiden Fällen zunächst vom YouTube-Kanal der Zeitung Die Welt bereitgestellten Videos berichtet. 
Wutreden“ (Tages-Anzeiger online, 3.2.2015), so wird dieser Trend von einer Online-Zeitung selbst auf den Punkt gebracht. Insgesamt lässt sich also seit etwa zehn Jahren im öffentlichen Diskurs eine zunehmende Subsumierung verschiedenster Redeereignisse unter diese Bezeichnung und mithin eine Extensionserweiterung sowie generell eine Zunahme der Verwendungshäufigkeit des Ausdrucks beobachten. ${ }^{5}$

\section{Die Wutrede als Gattung?}

\subsection{Zum Gattungsbegriff}

Inwiefern lässt sich nun dieser besondere Redetypus, für den sich in den letzten Jahren die Bezeichnung Wutrede etabliert hat, als Gattung beschreiben? Schließlich fallen hierunter Redeereignisse verschiedenster Redeanlässe und -situationen, angefangen von Pressekonferenzen über parlamentarische und andere politische Reden bis hin zu (Telefon-)Interviews. In traditionell orientierten rhetorischen Gattungstypologien findet die Wutrede schon aus diesem Grund

5 Mit Blick auf die ausdrucksseitige Gestalt des Wortes Wutrede könnten natürlich Verbindungen gezogen werden zwischen der Konjunktur dieses Wortes in den letzten Jahren einerseits und der Wahl des Wortes Wutbürger zum Wort des Jahres 2010 andererseits, die Journalisten längst zur Neuschöpfung einer ganzen Reihe von analogen Komposita inspiriert haben dürfte. Im DeReKo lassen sich nach 2010 etwa Belege für Komposita wie Wutbauer, Wutgriechen, Wutmieter, Wutpolitiker, Wutsenioren und sogar Wutwissenschaftler finden, mit denen in Analogie zu den „aus Enttäuschung über bestimmte politische Entscheidungen sehr heftig öffentlich protestierende[n] und demonstrierende[n] Bürger[n]“ (Duden) auf Protestbewegungen aller Art Bezug genommen wird. Ebenso finden sich seit 2010 vermehrt Komposita mit dem Determinans Wut-, mit denen auf diverse Verlautbarungen aus dem Impetus des Protests referiert werden kann, wie etwa Wut-Anträge, Wut-Demo, Wut-Mail, Wutbrief oder Wutvotum. Schließlich finden sich Komposita wie Wuttage, Wutwelle oder Wutvirus, die in abstrakter und häufig metaphorischer Weise auf die Protestbewegungen Bezug nehmen und mithin weitläufige soziale Prozesse auf das Moment der Wut zu reduzieren scheinen. Es spricht einiges dafür, die Konjunktur des Wortes Wutrede und die sich hierin zeigende Extensionserweiterung als ,Windschatteneffekt ${ }^{*}$ der Ausbildung eines ganzen Wortbildungsnests (vgl. Fleischer/Barz 1995: 71) anzusehen. Das Lexem Wut weist offenbar eine hohe Wortbildungsaktivität auf und wird zudem, anders als bei seit langem usuellen Komposita wie Wutanfall, Wutausbruch oder Wutgeheul, auch insgesamt semantisch unspezifischer: Es referiert nicht mehr nur auf Wut als Basisemotion im Sinne einer intern repräsentierten, subjektiv erfahrbaren Evaluationskategorie, die sich auf bestimmte Weise im Ausdrucksverhalten manifestiert (vgl. Schwarz-Friesel 2013: 73). Wut verweist vielmehr auch auf ein umfassenderes Konglomerat an Praktiken der Kritik und des Protests, die ihrerseits verstärkt als emotionsbasiert konzeptualisiert werden. 
keinen rechten Platz. Die hier etablierte polemische Redegattung der Invektive (Schmährede), die sich der rhetorischen Lehre entsprechend durch die Redeabsicht der Herabsetzung einer öffentlichen Person definiert und sich aus subjektiv empfundenen Affekten wie Hass und Wut speist (vgl. Neumann 1998: 549551), deckt sich nicht mit der Wutrede im heutigen Sinne. Die Wutrede als spontan-impulsiver Ausbruch, der zudem nicht auf die klassische Situation der monologischen Rede vor einem bloß zuhörenden Publikum beschränkt ist, wird von der klassischen Redegattungslehre, die sich auf im Sinne der fünf Produktionsstadien wohlvorbereitete und -vorgetragene Reden konzentriert, gar nicht erst berücksichtigt. Auch der in der klassischen Rhetorik vielfach reflektierte Jähzorn (iracundia) hat nur insofern rhetorische Relevanz, als er im Sinne beabsichtigter Entrüstung zu persuasiven Zwecken gezielt eingesetzt wird (vgl. Pichl 1998: 597). Hier wäre die (vereinzelt mit Wutrede synonym gesetzte) Brandrede zu nennen, die als Rede anklägerischen Inhalts (Duden) aufrüttelnde Funktion hat. Nur ein Teil der Wutreden ist hierdurch adäquat charakterisiert.

Neuere Gattungstheorien sind demgegenüber wesentlich offener angelegt und bestimmen Gattungen ähnlich wie Textsorten als ,familienähnliche' (vgl. Wittgenstein 1984: 278) Gruppen von Texten, denen formale, inhaltliche oder auch funktionale Texteigenschaften gemeinsam sind (vgl. zusammenfassend Reisigl 2014: 158). Vor allem die soziologisch orientierte Gattungsforschung hat - angeregt etwa durch Bakhtins (1986) Begriff der Redegattung (speech genre) oder durch die Arbeiten zur Ethnographie der Kommunikation - den Gattungsbegriff so erweitert, dass er nicht länger auf schriftliche, insbesondere literarische Formen oder auf (auch mündlich tradierte und vorgetragene) Kunst(rede)gattungen beschränkt ist. Gerade auch die alltäglichen Gattungen, die als Ethnokategorien im Wissen von Kommunikationsgemeinschaften verankert sind, ohne in kodifizierter Form (etwa in Gattungspoetiken) geregelt zu sein, wurden als „kommunikative Gattungen“ der Gattungsforschung zugänglich gemacht (vgl. Günthner/Knoblauch 1994: 696-699).

Ungeachtet der Besonderheiten, die kommunikativen Gattungen gegenüber Textsorten im konventionellen Sinn zugeschrieben werden (wie etwa die zentrale Rolle interaktiver Aspekte) und die die Gattungsforschung von herkömmlicher Textsortenlinguistik unterscheiden (vgl. hierzu Günthner 1995: 208-211), sind kommunikative Gattungen als verfestigte Muster natürlich auch in ihren formalen und inhaltlichen Eigenschaften zu beschreiben. Diese der sog. Binnenstruktur zuzuweisenden Eigenschaften unterschiedlicher Reichweite werden in der Analyse durch sozialstrukturelle Anbindungen (Außenstruktur) und durch typische situativ bestimmte Handlungsmuster (Zwischenebene) ergänzt. Dabei werden Gattungen als prototypisch strukturiert gedacht, und nicht alle Merkmale sind 
von gleichem Gewicht für die Zugehörigkeit eines Exemplars zu einer Gattung (vgl. auch Hanks 1987: 681).

So gefasst kann nun auch die Wutrede tentativ als Gattung bestimmt werden. Die sich in der längst etablierten Bezeichnung Wutrede manifestierende Ethnokategorie eines bestimmten Redetypus, aber auch das sich in diversen Rezeptionszeugnissen niederschlagende Wissen um typische Merkmale von Wutreden (s. $\mathrm{u}$. Kap. 4) sind Anlass genug, die Wutrede einmal probeweise im Sinne der Theorie der kommunikativen Gattungen näher zu charakterisieren. Gerade für die Beschreibung mündlicher Kommunikationstypen hält die Theorie der kommunikativen Gattungen ein überaus differenziertes Instrumentarium bereit und hat nicht zuletzt wegen ihrer Unterscheidung der drei genannten Strukturebenen einen hohen heuristischen Wert. Wie jedoch zu zeigen sein wird, greift sie am besonderen Fall der Wutrede als einer vornehmlich rezeptiven Typisierung vorbei. Getreu dem Prinzip der hermeneutischen Billigkeit (vgl. Meier 1757: 20) gilt es aber, die Theorie zunächst so stark wie möglich zu machen, um anschließend umso besser ihre ,blinden Flecken` zeigen zu können.

\subsection{Gattungsmerkmale von Wutreden - empirisch betrachtet}

Zur Erfassung der typischen Merkmale von Wutreden habe ich, zusätzlich zu den bisher zu Rate gezogenen Presseartikeln, ein Datenkorpus von insgesamt 30 Wutreden erstellt, die im Internet als Videoaufzeichnungen ${ }^{6}$ verfügbar sind, und Transkriptionen angefertigt. Die Recherche erfolgte tentativ durch verschiedene Suchanfragen und -strategien, angefangen von der Suche nach dem Schlagwort „Wutrede“ auf der Videoplattform YouTube und der Videosuche mit Google bis hin zur gezielten Suche nach Aufzeichnungen von Redeereignissen, über die in den Medien ausdrücklich als Wutreden berichtet wurde. Bedingung für die Aufnahme eines Videos in das Korpus war also, dass in den Videoüber- oder -unterschriften oder auch anderen Begleittexten wie Tags einschließlich Medienberichten auf das Video bzw. das dort gezeigte Redeereignis ${ }^{7}$ mit dem Ausdruck Wutrede Bezug genommen wird. Dass man auf diesem Wege auf Redeereignisse stößt, die man ohne diese ausdrückliche Bezeichnung womöglich nicht als Wutrede einschätzen würde, ist ein Faktum, das für mich aber weniger ein methodisches Hindernis als

6 Eine Ausnahme stellt das Radiointerview mit dem Musiker Sven Regener im Bayerischen Rundfunk vom 25.3.2012 dar, das nur als Audioaufzeichnung verfügbar ist.

7 Wie noch zu zeigen sein wird, ist in vielen Fällen gerade für die Gattungsproblematik zwischen den (oftmals geschnittenen) Videos und den Redeereignissen selbst zu unterscheiden. 
vielmehr ein zu erklärender Befund ist. Um aber die nicht $\mathrm{zu}$ übersehende Willkürlichkeit in den vorfindlichen Videobezeichnungen abzufangen, wurde einerseits auf diejenigen Wutreden besonderes Augenmerk gerichtet, die in der Rezeption - sprachlich markiert etwa durch das Attribut legendär - zu Idealtypen stilisiert werden (vgl. Voßkamp 1997: 655f.). Andererseits wurden Videofunde mit dem öffentlichen Diskurs in den redaktionellen wie auch den sozialen Medien abgeglichen und nur dann berücksichtigt, wenn über das Redeereignis auch andernorts als „Wutrede“ gesprochen wurde. Eine gewisse überindividuelle Übereinstimmung in der Beurteilung der Rede als Wutrede wurde also sichergestellt.

15 der 30 Wutreden wurden von Fußballtrainern, weitere 8 von anderen Akteuren des Profifußballs (Funktionäre, TV-Experten etc.) gehalten. Die verbleibenden Wutreden fallen auf Künstler (4) und Politiker (3). Die Aufzeichnungen im Datenkorpus sind zwischen 38 Sekunden und 25 Minuten, im Schnitt 4:17 min lang. ${ }^{8}$ Insgesamt umfasst das Korpus rund 2:10 Stunden Material. Die Transkripte wurden nach den GAT2-Konventionen für Basistranskripte erstellt und umfassen rund 20000 Wörter. Nonverbale Handlungen und Ereignisse wurden nicht eigens notiert, allerdings erfolgte die Analyse der Transkripte immer im Verbund mit den entsprechenden Videodateien.

Mit Blick auf das Korpus lässt sich die prototypische Wutrede nun den Analyseebenen der Theorie der kommunikativen Gattungen entsprechend wie folgt näher bestimmen:

Hinsichtlich der Außenstruktur der Wutrede ist zuallererst die (mediale) Öffentlichkeit der Redesituation zu nennen. Die Wutredner sind als Funktionsträger öffentliche Personen, die ihre Reden vor der Presseöffentlichkeit halten und mitunter, wie im Fall von Pressekonferenzen, auch primär an diese adressieren. Die Teilnehmerkonstellation entspricht ganz den Strukturen der massenmedialen Kommunikation: Auch wenn die Redner ihre in der Wutrede artikulierte Kritik häufig an die unmittelbar Anwesenden richten (z. B. an die teilnehmenden Journalisten bei einer Pressekonferenz), müssen sie sich des erweiterten Rezipientenkreises und der entsprechenden Mehrfachadressierung gewahr sein. ${ }^{9}$ Darüber hinaus erweist sich die Wutrede empirisch betrachtet als geschlechtsspezifisch. In meinem Datenkorpus finden sich ausschließlich Videos von männ-

8 Man beachte auch hier, dass es sich bei den Videos zumeist um Ausschnitte, oft sogar um Zusammenschnitte handelt.

9 Allerdings sind vereinzelt auch nichtöffentliche Wutausbrüche als Wutreden bezeichnet worden. So wurde der Fußballtrainer Claus-Dieter Wollitz heimlich mit einer Handykamera gefilmt, als er im Gespräch mit Fans auf dem Vereinsgelände in Wut geriet und dabei die Vereinsführung heftig kritisierte. Über das Video wurde in der Presse ausführlich als „Wutrede“ berichtet. 
lichen Rednern, aber auch in der medialen Berichterstattung kommen Wutrednerinnen nur äußerst selten vor.

Im Hinblick auf die Binnenstruktur der Wutrede lassen sich zunächst inhaltliche Verfestigungen feststellen. Wutreden sind häufig impulsive Reaktionen auf explizite wie implizite Kritik an der Arbeit des Redners oder auf andere als unangebracht erachtete Verhaltensweisen. Ganz im Sinne klassischer Theorien der Wut bzw. des Zorns aus der Rhetorik wie auch der neueren kognitivistischen Emotionsforschung, die Wut als eine Form der negativen Bewertung von Handlungen, Ereignissen und Sachverhalten definieren (vgl. Hodapp 2000: 201f.), thematisieren die Wutredner häufig die Anlässe für ihre Wut, die sie als unangemessen und herabsetzend kennzeichnen und mithin ihrerseits kritisieren (Bsp. 4 und 5).$^{10}$ Hinsichtlich des Redegegenstands sind Wutreden also gewissermaßen diskursive Explikationen einer emotionalen Szene (vgl. Wierzbicka 1999: 88; Fries 2003: 120).

(4) ich kann gewisse dinge nicht akzeptieren, wenn nen trainer wie der letzte DEPP dargestellt wird, (.) als hätt er GAR keine ahnung; (Bruno Labbadia, Fußballtrainer, Pressekonferenz, 8.10.2012)

(5) und DAS is wichtig für mich; dass wir DA mal die konzentration drauflegen; und nicht um um äh äh äh ob ICH noch in zwei wochen da bin; oder ob ob ob ob DER noch nächste saison hier fußball spiel=das is re!SPEKT!los (.) ohne ende. (Thomas Doll, Fußballtrainer, Pressekonferenz, 23.4.2008)

Mitunter nehmen die Wutredner auf die eigene Wut ausdrücklich mit emotionsbezeichnenden Lexemen (vgl. Schwarz-Friesel 2013: 144) Bezug, wobei vornehmlich das Verb ärgern vorkommt:

(6) und !DAS! is das was mich so ärgert. (Thomas Doll, Fußballtrainer, Pressekonferenz, 23.4.2008)

Es überwiegen jedoch bei weitem Phänomene des Emotionsausdrucks (vgl. Schwarz-Friesel 2013: 151), also verbale, para- und nonverbale Manifestationsformen emotionaler Einstellungen, die bereits in einer Reihe von gesprächsanalyti-

10 Schon bei Aristoteles findet sich diese Sichtweise, wenn Zorn definiert wird als „ein mit Schmerz verbundenes Streben nach einer vermeintlichen Vergeltung [...] für eine vermeintliche Herabsetzung einem selbst oder den Seinigen gegenüber, denen eine Herabsetzung nicht zusteht“ (Aristoteles Rhet 1378a). Dies kehrt auch in neueren kognitionslinguistischen Arbeiten zu Emotionskonzepten wieder. So bestimmt etwa Kövecses (1990: 67f.) das prototypische Szenario für Ärger wie folgt: „There is a wrongdoer who intentionally does something directly to $S$. The wrongdoer is at fault und $S$ is innocent. The offending event constitutes an injustice and produces anger in S.“ Dabei gilt ärgerliches Verhalten als „act of retribution“. 
schen Arbeiten beschrieben worden sind. So hat Spiegel (2011: 231f.) für Streitgespräche Manifestationen der emotionalen Beteiligung beschrieben, die in ähnlicher Form auch bei Wutreden zu beobachten sind. Wenngleich diese zumeist monologisch und mithin keine typischen Streitgespräche sind, findet sich auch hier der von Spiegel erwähnte Registerwechsel hin zu einer niedrigeren Stilebene mit derbem, negativ bewertungshaltigen Schimpfvokabular (Bsp. 7 und 8) und vermehrten dialektalen Anteilen (Bsp. 9):

(7) da lach ich mir doch n ARSCH ab (Thomas Doll, Fußballtrainer, Pressekonferenz, 23.4.2008)

(8) das is kein geschäftsmodell das is scheiße ja? (Sven Regener, Musiker, Telefoninterview, 25.3.2012)

(9) wat wolln se (Per Mertesacker, Fußballspieler, Interview, 30.7.2013)

Ebenfalls kennzeichnend für die niedrigere Stilebene, zu der die Sprecher häufig erst im Verlauf ihrer Rede - gewissermaßen im Zuge des Sich-in-Rage-Redens übergehen, sind nähesprachliche Anredeformen wie du, $i h r^{11}$ oder auch Spitznamen wie Waldi:

(10) eure scheiß stimmung da seid IHR doch dafür verantwortlich und nicht wir (Uli Hoeneß, Fußballmanager, Vollversammlung des Vereins, 13.11.2007)

(11) ihr solltet euch überlegen wer hier die kriegstreiber sind (Frank-Walter Steinmeier, Politiker, Kundgebung, 20.5.2014)

(12) die ISländer sind taBELLenführer das weisst du waldi (Rudi Völler, Fußballtrainer, TV-Interview, 6.9.2013)

Weitere oberflächensprachliche Merkmale, in denen eine als Wut zu deutende emotionale Beteiligung des Sprechers zum Ausdruck kommt, sind Wiederholungen von einzelnen Lexemen oder auch Satzkonstruktionen - Fiehler (1993, 161) nennt dies ,insistierende[s] Iterieren“ - zur Intensivierung der eigenen Aussage (vgl. auch Spiegel 2011, 244):

(13) das isch hh das isch (.) schlimm (-) schlimm (.) die berlingschichte isch schlimm (Christian Streich, Fußballtrainer, Pressekonferenz, 26.8.2013)

(14) das is doch alles bla bla bla is das doch; (-) ALLES bla bla bla is das. (Thomas Doll, Fußballtrainer, Pressekonferenz, 23.4.2008)

(15) WER hat dann für unruhe gesorgt; ICH hab dann für unruhe gesorgt? ich HABE nicht für unruhe gesorgt. (Claus-Dieter Wollitz, Fußballtrainer, Gespräch mit Fans auf dem Vereinsgelände, 13.5.2013)

11 Zum emotionsausdrückenden Potential der du-Anrede vgl. Zifonun/Hoffmann/Strecker (1997: 925). 
Häufig finden sich auch Generalisierungen und hyperbolische Ausdrucksweisen, insbesondere bei kritischen Bezugnahmen auf die wutauslösenden Handlungen, Ereignisse und Sachverhalte:

(16) und dann wir dir JEDES MAL werden dir knüppel irgendwo reingeworfen. (Thomas Doll, Fußballtrainer, Pressekonferenz, 23.4.2008)

(17) IMmer dieses dieses diese geschichte alles in in den !DRECK! ziehn alles RUNterzuziehn; das is das allerLETZte; (Rudi Völler, Fußballtrainer, TVInterview, 6.9.2003)

Ebenfalls bei kritischen Bezugnahmen auf die Wutanlässe findet sich oftmals eine spezifische Form der stilisierenden, prosodisch auffällig markierten Redewiedergabe, die es dem Sprecher erlaubt, die porträtierte Rede als unangemessen darzustellen und mithin auch die wiedergegebenen Sprecher negativ zu bewerten (vgl. hierzu Günthner 2002: 63):

(18) und bringen jetz als hilfstruppen die ganzen DEPPEN ins spiel die sagen 个warum kann ich denn das video nich auf youtube gucken $\downarrow$ ja dann kucks halt woANDers (Sven Regener, Musiker, Telefoninterview, 25.3.2012)

Die den Gegnern in den Mund gelegte Frage „warum kann ich denn das video nich auf youtube gucken“ wird von Regener in deutlich höherer Frequenz gesprochen, stellt den zitierten Sprecher als dümmlich dar und lädt, insbesondere in der Interviewsituation als einem Gespräch vor Publikum, „die Rezipient/inn/en zur gemeinsamen Verurteilung des porträtierten Verhaltens ein“ (Günthner 2002: 66). Wut als „Streben nach einer vermeintlichen Vergeltung“ (Aristoteles Rhet 1378a) findet in solchen Strategien der Verurteilung ihren Ausdruck und ihr Ziel.

Schließlich finden sich auffallend häufig formelhafte konversationelle Strategiesignale („gambits“ (Keller 1981)), mit denen die Sprecher ihre eigene Rede auf spezifische Weise metakommunikativ rahmen (vgl. Hagemann 1997: 157): ${ }^{12}$

(19) am ARSCH geleckt (-) das muss ich hier mal GANZ klar deutlich SAgen; (Bruno Labbadia, Fußballtrainer, Pressekonferenz, 8.10.2012)

(20) ich kann diesen scheissdreck nich mehr HÖRN muss ich ganz ehrlich sagen; (Rudi Völler, Rudi Völler, Fußballtrainer, TV-Interview, 6.9.2003)

(21) das MUSSte ich jetzt einmal sagen. (Peter Kulka, Architekt, offizielle Eröffnung des Potsdamer Stadtschlosses, 19.2.2013)

Mit derartigen, insgesamt 38 mal vorkommenden Formeln verdeutlichen die Sprecher - ausdrücklich markiert durch das Modalverb müssen (vgl. hierzu

12 Für Hinweise zum Thema danke ich Sven Staffeldt. 
Niehüser 1987: 188-191) - die Dringlichkeit des vorgebrachten Anliegens und rechtfertigen zugleich die Normverletzung, welche die unverblümt vorgetragene Kritik und die derbe Ausdrucksweise darstellen. Wut kann „Motor einer Strategie der schonungslosen Offenlegung“ (Fiehler 2009: 769) sein, die hier jedoch nicht als selbst gewählte Strategie, sondern als nunmehr erforderliche Reaktion dargestellt wird (vgl. Stein 1995: 239; Imo 2007: 119). In einigen Fällen werden diese Formeln kombiniert mit dem Verweis auf eine nunmehr überschrittene ,Grenze، (vgl. hierzu Kövecses 1990: 67), was dem Redner keine andere Wahl lasse:

(22) da muss ich sage (1.0) aso DES isch natürlich WEIT über die grenze. (Christian Streich, Fußballtrainer, Pressekonferenz, 26.8.2013)

(23) es is ne toTALe grenze erreicht; (-) auch HIER in stuttgart des muss ich ihnen GANZ klar sagen; (Bruno Labbadia, Fußballtrainer, Pressekonferenz, 8.10.2012) $)^{13}$

Neben all diesen sprachlichen Phänomenen lassen sich auch typische paraverbale Merkmale aufzeigen, welche die Reden bzw. Redebeiträge als Ausdruck von Wut erkennen lassen. Neben der erhöhten Sprechlautstärke und der gepressten Stimme ist hier etwa die für den Ausdruck von Wut typische überdeutliche Artikulation (vgl. Sendlmeier 2012: 106) zu nennen:

(24) das is re!SPEKT!los (.) ohne ende (Thomas Doll, Fußballtrainer, Pressekonferenz, 23.4.2008)

Bei den typischen nonverbalen Merkmalen lässt sich das heftige, ausladende Gestikulieren nennen sowie ein Gesichtsausdruck, der in der psychologischen Literatur als „Ärgermimik“ (Hodapp 2000: 200) beschrieben ist.

Zuletzt lassen sich auch auf der situativen Realisierungsebene Musterhaftigkeiten nachweisen. Wutreden sind tendenziell monologisch, und zwar auch im Rahmen von eher dialogischen Kommunikationsformaten wie Interviews. Diese können insbesondere dann zu Wutreden werden, wenn die üblichen Mechanismen des Sprecherwechsels einseitig suspendiert werden und der Sprecher gleichsam in einem Redeschwall seine Meinung kundtut. ${ }^{14}$ Besonders deutlich wird dies bei einer als Wutrede bekannten, nur 44 Sekunden dauernden Pressekonferenz des Fußballtrainers Klaus Augenthaler, in der er die Fragen selbst stellt

13 Beide Redner beziehen sich hierbei auf die Journalisten, die mit ihrer kritischen und skandalisierenden Berichterstattung die Grenzen des ,Schicklichen` überschritten hätten.

14 Dies macht etwa der Musiker Sven Regener explizit, wenn er sich bei seinem Radiointerviewpartner noch während seiner Wutrede dafür entschuldigt, „dass sie jetz so schwallartig hier runterkommen“ (die sprachliche Konstruktion ist unklar, sie bezieht sich offenbar auf den Interviewpartner, auf den der Redeschwall ,niederkommt'). 
und auch selbst beantwortet. Die ruhig vorgetragenen Fragen und Antworten selbst sind unauffällig und entsprechen dem erwartbaren Jargon, doch allein die Weigerung, Journalistenfragen anzunehmen, führte in der Rezeption zur Einordung als Wutrede.

Außerdem zeichnen sich Wutreden durch ein bestimmtes Beteiligungsformat (vgl. Günthner/Knoblauch 1994: 709f.) aus, das schon in der oben erwähnten Strategie der Offenlegung (vgl. Fiehler 2009: 769) angelegt ist. Wutredner verlassen oft ihre Rolle als Funktionsträger und sprechen mithin auch nicht mehr nur als Sprachrohr der durch sie vertretenen Institution. Diesen Rollenwechsel, der sich sprachlich auch in dem beschriebenen Registerwechsel zeigt, zeigen die Redner dabei oft auch selbst an. Der Trainer Bruno Labbadia eröffnet seine Wutrede mit der Ankündigung, „etwas in eigener Sache“ vorzubringen, und der Architekt Peter Kulka beschließt seine Wutrede mit dem Kommentar „es musste einfach mal raus“ sowie einer Entschuldigung beim Mäzen des Bauprojekts, dem er die Eröffnung des neu erbauten Stadtschlosses „vermiese“. Der Politiker Christian Lindner macht am Ende seiner als Wutrede bekannt gewordenen Reaktion auf einen Zwischenruf eine händereibende Geste mit dem Kommentar „so das hat Spaß gemacht“. Die Sprecher machen also oftmals deutlich, dass ihnen die aus den üblichen Routinen herausgehobene Stellung ihrer Reden durchaus bewusst ist.

Die prototypische Struktur der Gattung und die in sich abgestufte Relevanz der eben beschriebenen Gattungsmerkmale (vgl. Günthner/Knoblauch 1994: 705) für die Rezeption der Reden auch aus journalistischer Sicht lässt sich im Übrigen gut anhand der Berichterstattung über Wutreden ersehen. Von besonderer Bedeutung für die Gattungszugehörigkeit ist der Redegegenstand. Das Üben von Kritik an vermeintlichem Fehlverhalten scheint eine Bedingung dafür zu sein, dass die Rede als Wutrede bezeichnet wird. Neben dem Lexem Kritik bzw. kritisieren (Bsp. 25) selbst kommen in den Berichten häufig auch paraphrasierende Ausdrücke wie Rundumschlag (Bsp. 26), abrechnen oder kein gutes Haar an jmdm. lassen (Bsp. 27) vor:

(25) St. Paulis Trainer muss dem DFB-Kontrollausschuss seine Wutrede nach der Niederlage gegen Fürth erklären. In dieser hatte Lienen Schiedsrichter Petersen heftig kritisiert. (Hamburger Abendblatt Online, 18.2.2015)

(26) Labbadia-Wutrede nach 2:2 - Rundumschlag des VfB-Trainers (Bremer Nachrichten, 8.10.2012)

(27) In einer Wutrede rechnete der Gastgeber mit der Politik ab. Und ließ kein gutes Haar an der Klimaschutz-PR des Senats. (Hamburger Morgenpost, 12.8.2010) 
Selbst wohlvorbereitete und ruhig vorgetragene Reden werden in der Berichterstattung mitunter als Wutreden bezeichnet, sofern in ihnen mehr oder minder deutliche Kritik geübt wird. ${ }^{15}$

Die in den Wutreden zum Ausdruck gebrachte emotionale Beteiligung wird freilich auch oft hervorgehoben. Ausraster oder auch der Phraseologismus jmdm. platzt der Kragen sind typische Paraphrasen, die jedoch meist pauschal bleiben und nicht näher angeben, worin sich die Wut sprachlich zeigt (,redet sich in Rage“, Welt 3.2.2015). Allenfalls das derbe Vokabular wird in Zitatform oftmals erwähnt und die entsprechenden Passagen können dann symbolhaft für die ganze Rede stehen (,,Mistkäsescheißdreck'-Rede“, Spiegel Online 20.4.2010). Ausführlich werden dagegen die para- und nonverbalen Anteile thematisiert („Mit hochrotem Kopf, hervorquellenden Augäpfeln, wild gestikulierend“, Berliner Zeitung, 7.10.2006).

\subsection{Argumente für einen rezeptionsorientierten Gattungsbegriff}

Man kann also, so lässt sich diese Zusammenschau musterhaft wiederkehrender Merkmale zusammenfassen, Wutreden als Bündelungen inhaltlicher, formaler und funktionaler Merkmale beschreiben, diese Merkmale den drei Strukturebenen kommunikativer Gattungen zuweisen und die Wutrede auf diese Weise kommunikationstypologisch erfassen. Zwar gibt es einzelne Exemplare, die nur wenige der beschriebenen Merkmale aufweisen und trotzdem als Wutreden bezeichnet werden, doch aus eben diesem Grund wird in der Theorie kommunikativer Gattungen - so wie übrigens in neueren textlinguistischen Arbeiten (Luginbühl 2014b: 29f.) und literaturwissenschaftlichen Gattungstheorien auch (vgl. Zymner 2010) - der Begriff des Prototyps bemüht. Auch der von Wittgenstein (1984: 278) geprägte und in neueren Gattungstheorien oft aufgegriffene (vgl. Hempfer 2010) Begriff der Familienähnlichkeit der in Typisierungsakten zusammengefassten Elemente kann dieses - sich generell bei Begriffsbildungen stellende - Problem abfangen. Die Art, wie und mit welchen lexikalischen Mitteln

15 So wurde etwa die Weihnachtsansprache 2014 von Papst Franziskus, in der er deutliche Kritik an der Kurie übte, in deutschen Medien als Wutrede bezeichnet. 
über Wutreden berichtet wird, macht zudem deutlich, welche Merkmale von Wutreden besonders wichtig sind und welche zwar rekurrent, aber gleichwohl weniger ausschlaggebend sind. ${ }^{16}$

Gleichwohl ergibt sich das grundlegende Problem, dass ein zentrales Bestimmungsmerkmal von Gattungen nach diesem Begriffsverständnis, nämlich „historisch und kulturell spezifische, gesellschaftlich verfestigte und formalisierte Lösungen kommunikativer Probleme“ (Günthner/Knoblauch 1994: 699) zu sein, auf Wutreden kaum angewendet werden kann. Diese funktionale Bestimmung, die, wie eingangs erwähnt, längst in die pragmatische Textsortenlinguistik Eingang gefunden hat (vgl. Fix 2008: 27f.; Hauser/Luginbühl 2015: 17), nimmt ihren Ausgang vom Zweck der jeweiligen Texte bzw. Redeereignisse, der wiederum an bestimmte Situationen gebunden ist: Gattungen werden ,in besonderen Interaktionssituationen als Lösungen eines wiederkehrenden Problems hervorgebracht“ (Günthner/Knoblauch 1994: 696). ${ }^{17}$ Entscheidend ist mithin ihre situative Funktionalität. Wie aber kann man bei den zumeist spontanen Ausbrüchen den Zweck der Redeereignisse in der Weise bestimmen, dass hierdurch die Identität der Gattung fixiert würde, wie das Problem umreißen, für das Wutreden routinehafte Lösungen darstellen, die „als ,Fertigprodukte“ zur Verfügung“ (Günthner/Knoblauch 1994: 696) stehen? Man bedenke auch die besonderen Rezeptionsbedingungen der medial verbreiteten Wutreden, die ja nicht nur die direkt adressierten Zuhörer vor Ort, sondern gerade auch die Sekundärrezipienten umfassen. Wie soll man vor diesem Hintergrund die Situation definieren, auf die Wutreden als zweckhafte Gebilde bezogen sind? Anders als kommunikative

16 In jüngster Zeit scheint sogar Mündlichkeit keine notwendige Bedingung mehr für die Kategorisierung als Wutrede zu sein. So wurde ein kritisch mit den eigenen Fans ins Gericht gehender Facebook-Post des Schauspielers Til Schweiger vom 9.8.2015 als Wutrede bezeichnet. Der Post weist allerdings die aus dem Schreiben in den neuen Medien bekannten stilistischen Merkmale zur Markierung emotionaler Beteiligung wie iterierte Ausrufezeichen auf und kann mithin als Produkt ,wütenden Schreibens‘ angesehen werden (vgl. http://www.purestars.de/ artikel/til-schweiger-wutrede-nach-gabriel-talk-so-atzt-der-tatort-star-auf-facebook_a9314/1 [3.9.2015]).

17 Diese Anbindung des Gattungsbegriffs an die Kategorien des Zwecks und der Situation findet sich auch in der neueren rhetorischen Gattungstheorie, wo Gattungen als „typified rhetorical actions based in recurrent situations“ (Miller 1984: 158, vgl. auch Devitt 2004: 12-19) definiert werden, sowie in Swales’ Ansatz der Gattungsanalyse, der „a shared set of communicative purposes“ (Swales 1992: 46) als zentrales Bestimmungsmerkmal von Gattungen ansetzt. Daran ändert sich auch nicht prinzipiell etwas durch den in der Theorie der kommunikativen Gattung so sehr betonten reflexiven Kontextbegriff, demzufolge die interaktive Produktion bestimmter Gattungen den Kontext konstruiert, in dem sie erst interpretierbar werden (vgl. Günthner 1995: 209). Der „Kontext“ ist nach dieser Auffassung in erster Linie die „Kommunikationssituation“. 
Gattungen im engeren Sinne, die als generalisierte Handlungsoptionen zum gesellschaftlichen Wissensvorrat gehören und als Orientierungsrahmen für die Produktion und Rezeption zur Verfügung stehen, ist die Wutrede eben primär eine rekonstruktive Kategorie.

In Erweiterung der klassischen Theorie kommunikativer Gattungen ist das Konzept der medialen Gattungen (vgl. Ayaß 2011) entwickelt worden, unter dessen Überschrift auch sog. Medienrekonstruktionen seitens der Rezipienten untersucht werden. Als „rekonstruktive Gattungen“ werden hier alle Muster bezeichnet, „mittels derer vergangene Erlebnisse und Erfahrungen in der Kommunikation mit anderen bearbeitet werden“ (Ulmer/Bergmann 1993: 82). Wenn aber dort das Reden über Medienereignisse fokussiert wird, so wird dieses Reden selbst als Gattung beschrieben, und dabei steht ebenfalls das Problem, „wofür diese Gattung eine Lösung ist“ (Ayaß 2011: 286), im Zentrum der Überlegungen. Für die hier interessierende Frage, wie sich die Wutrede selbst als Gattung konstituiert, stellt also auch das Konzept der medialen Gattungen nicht die geeigneten begrifflichen Mittel bereit.

Aus diesen Gründen soll hier für den besonderen Fall der Wutrede eine Schärfung des Gattungsbegriffs vorgeschlagen werden, die besonders das Moment der Rezeption betont. ${ }^{18}$ Wutreden werden - und hierin sind sie mit anderen rekonstruktiven Typisierungen wie „Skandalrede“ oder auch „Panikmache“ vergleichbar - zumeist erst im Nachhinein und unter spezifischen medialen Bedingungen zu Wutreden ,gemacht', mitunter zur Überraschung oder gar gegen den Willen der Redner selbst. ${ }^{19}$ Zur Bestimmung der Wutrede als Gattung sind daher nicht allein die Wutreden als situativ verankerte und funktional bestimmbare Kommunikationsereignisse selbst in den Blick zu nehmen, die ohnehin überaus disparat sind. Vielmehr müssen auch und gerade die Interpretationsleistungen berücksichtigt werden, wie sie in einer Vielzahl von Rezeptionszeugnissen ablesbar sind - Rezeptionszeugnisse, die sehr wohl für ein gewisses ,Gattungsbewusstsein'sprechen.

Grundzüge eines solchen rezeptionsorientierten Gattungsbegriffs sind bereits vor längerem in der rezeptionsästhetischen Literaturwissenschaft (Gattung als „vorkonstituierter Erwartungshorizont“ (Jauß 1977: 330), der das Verständnis des

18 Die Nützlichkeit des Konzepts der kommunikativen Gattungen für die Analyse anderer Kommunikationstypen wie etwa das Bewerbungsgespräch (vgl. Birkner 2001) wird damit nicht in Zweifel gezogen.

19 So zeigte sich Presseberichten zufolge Frank-Walter Steinmeier überrascht darüber, dass sein Auftritt bei einer Kundgebung als Wutrede in den sozialen Netzwerken so große Resonanz erfuhr (vgl. http://www.tagesspiegel.de/politik/wut-rede-des-aussenministers-steinmeier-sprecher-legtnach-unverschaemtheit/9917884.html [25.2.2015]). 
Publikums orientiert) sowie, thematisch einschlägiger, in der amerikanischen Linguistischen Anthropologie entwickelt worden. Gattungen sind Hanks (1987: 670) zufolge weniger als formal bestimmbare Gruppierungen thematischer und stilistischer Elemente anzusehen denn als „orienting frameworks, interpretive procedures, and sets of expectations that are not part of discourse structure, but of the ways actors relate to and use language.“ In Fortführung dieser Position nehmen Briggs und Bauman (1992) die intertextuellen Aspekte von Gattungen und Gattungszuschreibungen in den Blick: Wird ein Redeereignis einer bestimmten Gattung zugewiesen, wird dessen Rezeption durch seine (möglicherweise erst durch das aufnehmende Publikum konstruierten) intertextuellen Bezüge zu vorherigen Texten vermittelt und geprägt, so dass es in einen weit über sein unmittelbares situatives Setting hinausreichenden Rahmen gestellt wird (vgl. Briggs/ Bauman 1992: 147). Auf diese Weise wird ein situativ eingebetteter und angeschlossener Diskursausschnitt herausgehoben und als abgrenzbarer Text erfahrbar (vgl. auch Bauman/Briggs 1990: 73). ${ }^{20}$ Gattungszuschreibungen sind mithin als de- und rekontextualisierende Praktiken zu verstehen, durch die diskursive Ordnung in dem Sinne hergestellt wird, dass bestimmte Deutungsrahmen eingebracht werden (vgl. auch Spitzmüller 2013: 246). Im Ergebnis führt dies zu einem Gattungsbegriff „that places generic distinctions not within texts but in the practices used in creating intertextual relations with other bodies of discourse“ (Briggs/Bauman 1992: 163, Hervorh. S. M.). ${ }^{21}$

Gerade für den Fall von Wutreden als Redeereignissen, die erst durch die (von der ursprünglichen Redesituation abgelösten) Interpretationen als Exemplare von Wutreden erkennbar werden, bietet nun ein solcher Gattungsbegriff vielfaches Erklärungspotential. Denn insbesondere in den digitalen Medien als dem Ort, an dem Wutreden inzwischen vornehmlich rezipiert werden, sind die intertextualitätsstiftenden und gattungskonstituierenden Praktiken anhand einer Fülle von vielfältigen Rezeptionszeugnissen sichtbar und für die Analyse zugänglich. Zum rekonstruktiven Reden über die Medienereignisse, wie es auch in der Theorie der medialen Gattungen thematisiert wird, kommen hier noch die Rekon-

20 Vgl. hierzu auch den aus der neueren rhetorischen Gattungstheorie stammenden Begriff des „uptake“ (Freadman 2002: 40): Ein Text wird durch „taking it as“ einer bestimmten Gattung zugewiesen, hierdurch mit anderen Texten in Bezug gesetzt und so erst als Text mit einer bestimmten Funktionalität bestätigt (vgl. auch Giltrow/Stein 2009b: 6). Auch Beaugrande/ Dressler (1981: 12f.) setzen Intertextualität als Textualitätskriterium und mithin als Bedingung dafür an, dass eine kommunikative Okkurenz ein Text ist.

21 Es soll nicht unerwähnt bleiben, dass sich auch Günthner (1995: 195f.) auf die erwähnten Ansätze der linguistisch-anthropologischen Gattungstheorie beruft, dabei aber andere Aspekte ausschöpft. 
textualisierungen der entsprechenden Videos in neue, semiotisch komplexe Umgebungen hinzu. Die von Habscheid (2011a: 17) für eine rekonstruktive Kommunikationstypologie vorgeschlagene Untersuchung alltäglicher Reflexionspraktiken „im Rahmen von Performativität und Performanz“ findet hier einen vielversprechenden Gegenstand. Dies sei im Folgenden mit empirischen Belegen erläutert.

\section{Rekontextualisierung und Gattungskonstruktion in den (digitalen) Medien}

In vielen Kommentierungen von Wutreden, sowohl in der Berichterstattung über Wutreden als auch in Texten, welche die mediale Verbreitung der Videoaufzeichnungen flankieren, lassen sich verschiedene Arten von ,Gattungsreferenzen“ finden, also mehr oder minder explizite Bezugnahmen auf den Umstand, dass das betreffende Redeereignis ein Exemplar eines übergeordneten Typus darstellt. Schon die typisierende Benennung als Wutrede kann hier genannt werden, zeigt sich hierin doch eine beiordnende Kategorisierung einzelner Redeexemplare durch die Rezipienten. ${ }^{22}$ Heckenausdruckähnliche Attribute wie regelrecht (Bsp. 28), wahr (Bsp. 29 und 30) oder echt (Bsp. 31) verweisen auf prototypisch strukturiertes Gattungswissen und bringen darüber hinaus implizit Gattungsnormen ins Spiel, die eine ,echte' Wutrede bestimmen.

(28) Anfang Oktober ließ sich der für gewöhnlich smarte Deutsch-Italiener sogar zu einer regelrechten Wutrede wider seine Kritiker hinreißen (,Am Arsch geleckt“). (Nürnberger Nachrichten, 1.12.2012)

(29) Hoeneß hatte auf die Bemerkung eines Fans, die Stimmung in der AllianzArena sei schlecht, mit einer wahren Wutrede geantwortet. In dieser verurteilte er den Angriff auf die Vereinsführung als „populistische Scheiße“. (Spiegel Online, 15.11.2007)

(30) Ein hämischer Zwischenruf hat den FDP-Politiker Christian Lindner zu einer wahren Wutrede provoziert. Mit erhobenem Zeigefinger stürzte er sich auf seinen Kollegen von der SPD. (Focus online, 3.2.2015)

22 In verschiedenen textlinguistischen wie gattungstheoretischen Ansätzen wird daher das Textsortenwissen der Sprachbenutzer, wie es sich eben auch in der Benennungspraxis niederschlägt, zur Bestimmung von Textsorten und Gattungen herangezogen (vgl. etwa Miller 1984: 155, Swales 1992: 54, Günthner/Knoblauch 1994: 704). Durch ein solches rekonstruktives Vorgehen sollen die wissenschaftlichen Typologien an die tatsächliche Vielfalt textueller Wirklichkeit angeschlossen werden (vgl. zusammenfassend Habscheid 2011a: 14-17). 
(31) Im Europa-Wahlkampf zeigt sich Frank-Walter Steinmeier plötzlich von einer ganz anderen Seite. Den Protestrufen während einer Rede in Berlin begegnet der Bundesaußenminister mit einer so noch nie dagewesenen Emotionalität. Einer echten Wutrede. (Berliner Zeitung, 20.5.2014)

Belege dieser Art machen zudem erneut deutlich, dass die oben beschriebenen Merkmale wie derbes Vokabular oder das heftige Gestikulieren auch aus Rezipientensicht für die Frage der Gattungszugehörigkeit entscheidend sind.

Immer wieder werden aber auch die Wutreden ausdrücklich mit anderen Wutreden in Bezug gesetzt, wobei gerne Trapattonis Wutrede als Vergleichspunkt herangezogen wird. „Gattuso macht den Trapattoni“23 - so betitelt ein Onlinefußballmagazin den Videomitschnitt einer Pressekonferenz des italienischen, inzwischen in Griechenland arbeitenden Trainers. Über einen anderen Trainer wird berichtet, „eine Wutrede in bester Trapattoni-Manier“ gehalten zu haben. ${ }^{24}$ Aber auch über andere Funktionsträger wie etwa den Industriemanager Mehdorn wird berichtet, eine „Wutrede im Landtag“ gehalten zu haben. Dabei „erinnerte Hartmut Mehdorn an Giovanni Trapattoni, der mit seiner Wutrede in die Sportgeschichte einging“ (Märkische Allgemeine, 27.2.2014). In meinem Korpus findet sich sogar ein Fall, in dem der Wutredner selbst eine derartige Gattungsreferenz vornimmt. Noch während und inmitten seines Wutausbruchs während einer Pressekonferenz schiebt der österreichische Fußballtrainer Dietmar Kühbauer ein: „des war a trapattoni jetz jo“. Wohl in der Vorahnung der kommenden Berichterstattung reiht er sich ausdrücklich in die auf Trapattoni zurückgehende Tradition ein und deklariert seinen Ausbruch als ein Exemplar (,a trapattoni“) einer hier metonymisch mit dem Namen des Begründers bezeichneten Gattung.

Eine andere Form des Vergleichs ist auch die Rede von der „Wutrede des Jahres“ (Hamburger Morgenpost, 19.10.2012). In jüngerer Zeit, da die Presseberichterstattung überhaupt zusehends in die digitalen Medien übergreift (vgl. Burger/ Luginbühl 2014: 451f.) und gerade die Artikel über Wutreden oft nur mehr Begleittexte $\mathrm{zu}$ den mitpublizierten Videos sind, finden sich zudem auch ganze Kompilationen von Wutreden. Ein Beispiel hierfür ist die im Zuge der Berichterstattung über Christian Lindners Wutrede im NRW-Landtag zusammengestellte Liste „Die zwölf besten Wutreden“, die die entsprechenden YouTube-Links gleich mitliefert

$23 \mathrm{http} / /$ www.11freunde.de/video/gattuso-macht-den-trapattoni 24.9.2014 [25.2.2015] 24 http://www.welt.de/newsticker/sport-news/article113883849/Bommers-Wutrede-Cottbusversinkt-im-Mittelmass.html [25.2.2015] 
und dabei auch ältere Ereignisse wie etwa ein Interview mit Klaus Kinksi rückwirkend $\mathrm{zu}$ Wutreden erklärt. ${ }^{25}$ Videoplattformen wie YouTube, auf die viele Onlinezeitungen zurückgreifen, die aber auch vielfach direkt zur Rezeption der entsprechenden Videos aufgerufen werden, liefern derartige Kompilationen durch die rechts vom Hauptfenster angezeigten ähnlichen Videos (s. Abb. 1) gleich mit. Auch ohne ausdrückliche Zusammenschnitte wie das hier angeführte Video „Die Größten AUSRASTER und Sprüche der Bundesliga“ drängt sich eine serielle Rezeption mehrerer Wutreden geradezu auf.

Nächstes Video
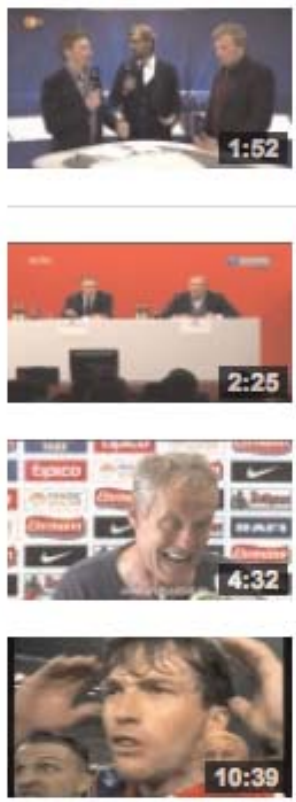

Autoplay

Jürgen Klopp rüffelt ZDF Reporter

von Sadie Douglas

136.747 Aufrufe

\section{Uli Hoeneß' Wutrede - TV BAYERN LIVE*} am 23.02.2014

von TVBayemlive

20.493 Aufrufe

\section{Christian Streichs Wutrede [26.08.13]}

von undFUSSBALL Clips

60.029 Aufrufe

Die Größten AUSRASTER und Sprüche der

Bundesliga

von Bundesliga Aktuell

Empfohlenes Video

Abb. 1: Ausschnitt aus youtube.com, „Wutrede“ Rudi Völler 29.11.2013, https://www.youtube. com/watch?v=vePAtOS_Fj0 [9.3.2015, 12:48 Uhr]

Aufschlussreich sind hierbei auch die angezeigten Thumbnails (Vorschaubilder), die einen beliebigen Ausschnitt gleichsam zur visuellen Überschrift machen und,

25 http://www.br.de/puls/themen/netz/wutrede-ausraster-fussball-politik-talkshow-best-of104.html [26.2.2015] 
wie im folgenden Fall (s. Abb. 2), für die Gattungsrelevanz der oben beschriebenen Ärgermimik sprechen:

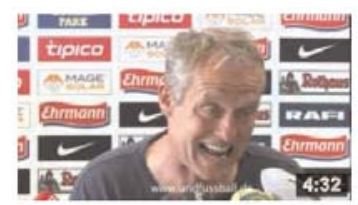

Christian Streichs Wutrede [26.08.13]

von undFUSSBALL Clips

vor 1 Jahr $\cdot 60.142$ Aufrufe

Christian Streich über seine Meinung zu dem Umgang der Medien mit der Entlassung von Bruno Labbadia und der Lolita-Affäre...

OFFIZIELL

Abb. 2: Ausschnitt aus youtube.com, https://www.youtube.com/results?search_query=streich +wutrede [9.3.2015, 12:55 Uhr]

Soziale Medien wie Twitter und Facebook laden durch hypertextuelle Vernetzungen mit Hashtags ebenfalls zu Reihenbildungen und entsprechend serieller Rezeption von Wutreden ein (s. Abb. 3 und 4):

Jan Kliewer $\otimes$ ChorinerStr667 $\cdot 4 \mathrm{~h}$

View translation 6 sz.de/1.2383286@SZ \#Wutrede Christof Kneer @ d \& \#Westermann \#Völler \#Trapattoni \#kleinerPissverein \#tarnfarbeneMaseratis

Abb. 3: Ausschnitt aus twitter.com, https://twitter.com/hashtag/wutrede [9.3.2015]

Andreas Krobok @akrobok - Feb 10

da hat der gute @c_lindner mit seiner \#wutrede wohl doch nicht ganz den richtigen erwischt m.faz.net/aktuell/politi...

ฐ FAZ.NET

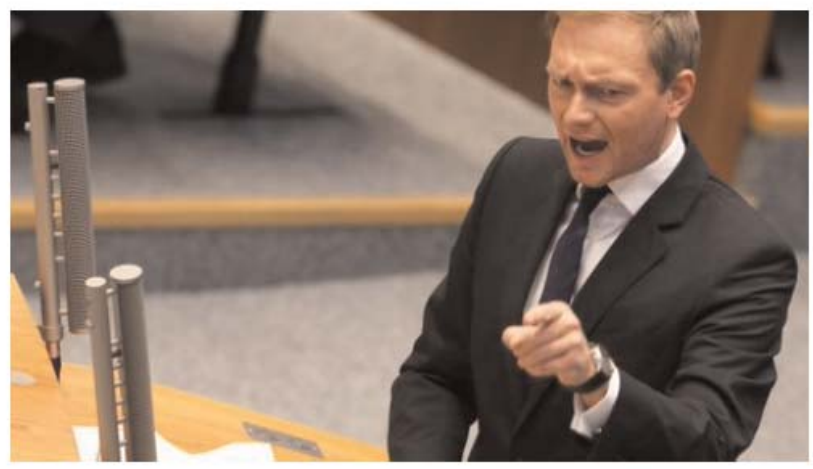

Christian Lindners Wutrede: Schutzherr der Gründungswilligen? By FAZ.NET @faznet

Mit einem Wutausbruch verteidigt Christian Lindner den Unternehmergeist und wird zum Internet-Star - doch die Sache hat mehrere Haken.

Abb. 4: Ausschnitt aus twitter.com, https://twitter.com/hashtag/wutrede [9.3.2015] 
Überhaupt zeigt sich, dass sich inzwischen Wutreden gerade über die sozialen Medien überaus rasch und, viral' verbreiten. Die herkömmliche Presseberichterstattung ist in vielen Fällen nur noch Reaktion auf den „Internet-Hit“, ${ }^{26}$ und die Pressemeldungen über diese Hits werden ihrerseits vielfach über Facebook und andere Dienste geteilt. ${ }^{27}$

Auf all diese Weisen werden also intertextuelle Bezüge zwischen einzelnen Exemplaren von Wutreden hergestellt. Diese werden zwar durch bestimmte, mehreren Exemplaren zukommende und mithin musterhafte Eigenschaften der betreffenden Redeereignisse angestoßen, wie sich etwa an den typischen sprachlichen Mitteln in der Berichterstattung über Wutreden sehen lässt. Dennoch sind diese intertextuellen Bezüge in erster Linie publikumsseitige Konstruktionen, welche die Redeereignisse aus ihren ursprünglichen Settings und ihrer situativen Funktionalität herauslösen und sie dadurch de- und wieder rekontextualisieren (vgl. Briggs/Bauman 1992: 164). Die Intentionen des Redners, aber auch die Wirkungen in der ursprünglichen Redesituation werden für die Rezeption letztlich irrelevant. Stattdessen rückt die Unterhaltungsfunktion in den Vordergrund, und ganz in diesem Sinne wird das überaus erfolgreiche Video der Wutrede von Frank-Walter Steinmeier auf dem YouTube-Kanal von Welt Online in die Kategorie „Unterhaltung“ eingeordnet. ${ }^{28}$

Für die Gattungskonstruktion bedeutsam und zugleich für die Analyse aufschlussreich sind auch die vielfach angebotenen Kommentarfunktionen der Onlinezeitungen und Newsportale wie auch der Videoplattformen selbst, über die sich die Mediennutzenden aktiv an den intertextualitätsstiftenden und mithin gattungskonstruktiven Diskursen über Wutreden beteiligen können. Neben den vielgestaltigen affirmativen Kommentierungen der Wutreden bezüglich Inhalt („Labbadia hat mehr als recht!“) oder auch der darin zum Ausdruck kommenden

26 http://www.faz.net/aktuell/politik/fdp-chef-keilt-aus-lindners-wutrede-wird-zum-internethit-13404895.html [15.9.2015]. Der Artikel über Lindners Wutrede wurde über 7000 mal via Facebook geteilt. Das Video wurde inzwischen rund über 500'000 mal, das Video der Wutrede von Steinmeier gar 2,7 Millionen mal aufgerufen.

27 Aus diesem Grund unterscheide ich hier nicht systematisch zwischen der professionelljournalistischen Berichterstattung über Wutreden einerseits und dem Laiendiskurs der Mediennutzenden andererseits. Das ,Publikum‘ von Wutreden umfasst Journalisten wie Mediennutzende gleichermaßen.

28 Der Unterhaltungscharakter von Wutreden, der auch mit einem gewissen medialen Voyeurismus zusammenspielen dürfte, ist wohl auch ein Grund dafür, dass Onlinemedien gerne das Schlagwort Wutrede verwenden, um Aufmerksamkeit zu erregen und letztlich die Zugriffszahlen zu erhöhen. Die Durchschaubarkeit dieses Vorgehens zeigt sich etwa im folgenden YouTubeKommentar: „also eine wutrede war das aber mal auch in keinster weise. klicksammler....“ (https://www.youtube.com/watch?v=vePAtOS_Fj0 [26.2.2015] 
Sprecherpersönlichkeit („Bruno Labbadia sollte für seine Ehrlichkeit belohnt werden!" $)^{29}$ kommen hier nämlich auch Äußerungen vor, mit denen Vorstellungen, Erwartungen oder Annahmen bezüglich Wutreden expliziert werden und die also die Gattungszugehörigkeit des jeweiligen Wutredenexemplars problematisieren (vgl. Gülich 1986: 22). So schreiben Leser auf Spiegel Online zum Artikel über Labbadias Wutrede: ${ }^{30}$

(i) Also eine Wutrede habe ich nicht gesehen. Wenn man das mit Trappatoni oder Völler nach dem Island-Länderspiel vergleicht, dann war das gar nichts! (schwanerich, SpOn, 8.10.2012)

(ii) Das soll eine Wutrede gewesen sein? Ich dachte ich bekomme einen „Ausraster“ a la Thomas Doll, Rudi Völler oder Trappatoni zu sehen. Die Ansprache werte ich eher als eine energische Zusammenfassung von Geschehnissen gepaart mit Medienschelte. (onecomment, SpOn, 8.10.2012)

Es finden sich auch ironische Kommentare, die in eine ähnliche Richtung zielen. So postet ein Fernsehsender auf seiner Facebookseite einen als Wutrede bezeichneten Mitschnitt aus einer Pressekonferenz des Hamburger SV, worauf sich zahlreiche Nutzer mit Kommentaren wie diesem hier zu Wort melden:

(iii) Oha was für ne „wutrede“ da zittert ja jeder Profi vor Angst :-D (Jan Zimmermann, Facebook, 12.12.2013) $)^{31}$

In solchen ironischen Kommentaren deutet sich hier die oben erwähnte Dominanz der Unterhaltungsfunktion von Wutreden an, die in vielen Kommentaren sogar explizit gemacht wird:

(iv) Solche Emotionen, will man doch sehen! KLASSE!! (Achim Peter, YouTube, ca. 2013) $)^{32}$

(v) Priceless, die Halsschlagader!! (Topocalma, Youtube, ca. 2014) 33

Schließlich sind hier auch die vielfachen spaßhaften Bearbeitungen der Wutredenvideos zu nennen. Viele Wutreden wurden mit Musik unterlegt und etwa zu

29 Beide Zitate im Kommentarbereich auf https://www.youtube.com/watch?v=73KsZbvyh_0 (20.3.2015)

$30 \mathrm{http} / /$ www.spiegel.de/sport/fussball/vfb-stuttgart-bruno-labbadia-haelt-eine-wutrede-a859974.html [26.2.2015]

31 https://www.facebook.com/SPORT1News/posts/10152037789659172 [26.2.2015]

32 https://www.youtube.com/watch?v=c9HqKTKPCng [26.2.2015]

33 https://www.youtube.com/watch?v=SqzWrEgwlyo [26.2.2015] 
Rapsongs umgestaltet. Ein als Wutrede bezeichnetes spielanschließendes Interview mit dem Fußballspieler Peer Mertesacker wurde gar parodistisch nachgestellt. ${ }^{34}$ Ausdrücklich als Parodien gibt es seit jüngstem auch bewusst als solche gehaltene Wutreden wie etwa ein vielfach via YouTube und Twitter verbreiteter Auftritt des Kabarettisten Roland Düringer im Österreichischen Fernsehen. ${ }^{35}$ Als wesenhaft intertextuelle Texte (vgl. Holthuis 1993: 118-123) sind solche Parodien in besonderer Weise gattungsindizierend. Aufschlussreich ist auch der Appell, den im November 2014 eine Onlinezeitung unter der Überschrift „Die längste Wutrede aller Zeiten“ an ihre Leser richtete: „Schicken Sie uns Ihre Wutrede als Audio-Datei und tragen Sie zur längsten Bahnstreik-Tirade der Geschichte bei.“36 Im November 2015 erschien in der Zeit schließlich sogar eine kritisch-polemische Glosse über das sogenannte Sabattical, die sich selbst im Untertitel ausdrücklich als „[e]ine Wutrede“ (Die Zeit, 5.11.2015) auswies. Diese Entwicklungen zeigen, dass die Gattungskonstruktion nunmehr an einem Punkt angelangt ist, wo die als typisch wahrgenommenen Merkmale von Wutreden ihrerseits handlungsleitend werden können - wenn auch zunächst nur im spielerischen Rahmen.

Als Erwartungshorizont und Orientierungsrahmen für die Rezeption und vereinzelt auch Produktion (vgl. Hanks 1987: 670), der die einzelnen Exemplare mit bereits bestehenden verbindet und Aussagen über Gattungszugehörigkeiten vonseiten der Diskursteilnehmer selbst ermöglicht, ist die Wutrede als Gattung also durchaus etabliert. Dieser Orientierungsrahmen ist jedoch keine direkte Folge der formalen und funktionalen Eigenschaften der ohnehin recht disparaten Redeereignisse. Als dynamische Größe bleibt er an die diskursiven Praktiken der De- und Rekontextualisierung gebunden, die, insbesondere in den digitalen Medien, als performative Verfahren (vgl. Habscheid 2011a: 17) sichtbar und beschreibbar sind. Gattungen, so formuliert Spitzmüller (2013: 246) in anderem Zusammenhang, ,sind genuin diskursive Phänomene, sie stellen diskursives Wissen dar und sind selbst diskursiv konstruiert (und mithin auch sozial und historisch veränderbar). “ Erst im anschließenden medialen Diskurs werden die als Wutreden bezeichneten und für sich genommen höchst unterschiedlichen

$34 \mathrm{http} / /$ www.focus.de/sport/videos/konfrontiert-mit-schlechtem-laktattest-profis-des-1-fckoeln-parodieren-mertesacker-mit-wut-interviews_id_3961160.html [27.2.2015]

35 http://wien.orf.at/news/stories/2513112/ [27.2.2015]

36 http://www.ruhrnachrichten.de/nachrichten/vermischtes/aktuelles_berichte/LaengsteWutrede-aller-Zeiten-Lassen-Sie-Ihre-Bahnstreik-Wut-gegen-GDL-oder-Bahn-heraus; art29854,2532563 [15.9.2015] 
Redeereignisse geordnet und vereinheitlicht, so dass auch Reihungen wie die angesprochenen Kompilierungen möglich werden. ${ }^{37}$

Wutreden, so lässt sich zusammenfassend festhalten, sind also stets und wesenhaft medial rekontextualisiert. Gerade die digitalen Medien mit ihren Verbreitungs- und auch Bearbeitungsmöglichkeiten von Videos und ihren Begleittexten sowie die dadurch mögliche zeitlich und örtlich entgrenzte Rezeption und Kommentierung liefern die Rahmenbedingungen (,affordances“ oder „Ermöglichungen“, vgl. Marx/Weidacher 2014: 57) für die gattungskonstruktiven Rekontextualisierungen. Es dürften also in erster Linie die besonderen Kommunikationsmodalitäten der digitalen und später auch der sozialen Medien mit ihren Kommunkationsmitteln wie Multimodalität, Hypertextualität und Interaktivität (vgl. Meier 2008: 238-279) gewesen sein, welche zu der eingangs aufgezeigten Konjunktur von Wutreden geführt haben - und nicht eine Zunahme von Redeereignissen als solchen, die die in Kap. 3.2 beschriebenen Merkmale von Wutreden aufweisen. Nicht zufällig scheint mir daher der sprunghafte Anstieg in den Erwähnungen von „Wutreden“ im Pressediskurs mit der technischen Innovation von YouTube und anderen Möglichkeiten der Implementierung von Videos in Internetseiten, aber auch der Entwicklung sozialer Medien zusammenzufallen. Öffentliche Wutausbrüche treffen in Zeiten des Web 2.0 auf gänzlich andere Rezeptions- und Interpretationsbedingungen und können so zu einer Gattung gerinnen, die deutliche diskursive Effekte zeitigt.

\section{Fazit}

Fragt man nach der Wutrede als ,Gattung“, als die sie wenigstens aus Sicht der Rezipierenden etabliert ist, so ist zu unterscheiden zwischen den ursprünglichen Redeereignissen in ihren jeweiligen situativen Kontexten einerseits und den medial rekontextualisierten „Wutreden“ andererseits. Eine Rede (oder auch nur ein Ausschnitt derselben) wird zur Wutrede gemacht, wenn sie als solche rezipiert und gerahmt wird. Die ursprüngliche, zweckhaft bestimmte und situativ verankerte Funktion der Rede wird dadurch womöglich vollständig suspendiert. Die spezifischen Kommunikationsmittel der digitalen Medien wie Multimodalität und Hypertextualität bieten für diese Rekontextualisierungen den geeigneten Rahmen.

37 Man bedenke auch, dass viele der mit „Wutrede“ überschriebenen Videos Zusammenschnitte sind. Die Langversion von Steinmeiers Wutrede auf dem Berliner Alexanderplatz ist ebenfalls als Video verfügbar, das mit rund 12000 Aufrufen nicht annähernd so erfolgreich ist wie die geschnittene Fassung mit ihren ca. 2,7 Mio. Aufrufen. 
Gerade mit Blick auf die Genese dieser Gattung wird hier also besonders der Rezeptionsaspekt von Gattungen betont. Als inhaltlich, formal und funktional bestimmbares Ensemble sprachlicher Merkmale ist die Gattung Wutrede noch nicht hinreichend bestimmt, und die Entstehung und Etablierung dieser Gattung ist allein mit Blick auf die wiederkehrenden kommunikativen Aufgaben, für die sie eine Lösung darstellen, noch nicht zu erklären. Die Gattung ist vielmehr als diskursiv konstruierter und diskursiv wirksamer Erwartungshorizont zu beschreiben, der die Redeereignisse auf eine bestimmte Weise ordnet und verbindet, sie dadurch vorstrukturierten Interpretationen zuführt und, das zeigen die jüngsten Entwicklungen, dann auch die Produktion oder wenigstens die spielerische Inszenierung neuer Exemplare möglich macht.

Auch wenn die Theorie der kommunikativen Gattungen wie auch verwandte Ansätze, die den routinisierten Problemlösungscharakter gattungsgebundenen Sprachhandelns betonen, gerade für mündliche Kommunikationstypen attraktiv und mit ihren feinkörnigen Beschreibungsmöglichkeiten heuristisch wertvoll ist, erscheint sie doch nicht dafür geeignet, die spezifischen Bedingungen der Emergenz von Typisierungen im Internet wie den hier beschriebenen adäquat zu erfassen. Dies liegt nicht allein am Status der Wutrede als vornehmlich rezeptive Typisierung, der wie oben erwähnt auch Kommunikationstypen wie der Skandalrede oder der Panikmache zukommt. Auch die spezifischen und durch die medialen Bedingungen wesentlich mitgeprägten Modi der rekonstruktiven Gattungszuschreibung, die sich auf rekontextualisierte Kommunikationsereignisse jenseits ihrer situativen Funktionalität richten, verlangen einen durchaus eigenständigen theoretischen und empirischen Zugriff.

Der als Wutrede bezeichnete Redetypus selbst, dies sei hier nochmals deutlich betont, stellt mitnichten ein neues Phänomen dar, denn öffentliche Wutausbrüche hat es natürlich schon vor Trapattonis initialer Wutrede ebenso gegeben wie metadiskursive Reflexionen über sie. Durchaus neu und auf neue Weise sichtbar sind dagegen die Arten, wie in medialen Kontexten die rekontextualisierten Redeereignisse seriell rezipierbar werden und wie auf sie in Anschlussdiskursen Bezug genommen wird. Diese Faktoren kommen sowohl in der Theorie der kommunikativen Gattungen, die sich auf die situative Funktionalität von typisierten Redeereignissen konzentriert, als auch in einer Textsortenlinguistik, die vornehmlich formale und funktionale Merkmale und deren Wandel fokussiert, tendenziell zu kurz. Denn, so formuliert Luginbühl (2014a: 308), ,it is important to see that the meaning of a genre can change due to changes in genre context, even if the form and the function of this genre do not seem to change." Gerade auch die Rezeptionsbedingungen und -praktiken machen diesen „genre context" maßgeblich aus. 
Eine diachrone Textsortenlinguistik bzw. Kommunikationstypologie, welche sich allein auf den Wandel sprachlicher Textmuster oder den Funktionswandel bestehender Textsorten beschränkt, bekäme darum ein Wandelphänomen wie die hier beschriebene Konjunktur von Wutreden, die sich durchaus in veränderter diskursiver Wirklichkeit niederschlägt und über einen bloßen Bedeutungswandel des Ausdrucks Wutrede hinausgeht, nicht in den Blick. In Zeiten des Web 2.0 ist aber ein solches Phänomen, das in medialer Rekontextualisierung gründet, kein randständiges mehr. ${ }^{38}$ Das Internet bringt eben auch veränderte Bedingungen und Formen der Rezeption hervor. Bei der Frage nach medial bedingtem Textsortenwandel sind auch solche Veränderungen von großer Bedeutung.

\section{Literatur}

Androutsopoulos, Jannis K. (2015): „Mediatisierte Praktiken. Zur Rekontextualisierung von Rezeptionskommunikation in den sozialen Medien“. Vortrag auf 51. Jahrestagung des Instituts für deutsche Sprache „Sprachliche und kommunikative Praktiken“, Mannheim, 10-12.3.2015.

Aristoteles (2002): Rhetorik. Werke in deutscher Übersetzung Bd. 4. Übers. u. erl. v. Christof Rapp. Berlin: Akademie-Verlag.

Ayaß, Ruth (2011): „Kommunikative Gattungen, mediale Gattungen“. In: Stephan Habscheid (Hg.): Textsorten, Handlungsmuster, Oberflächen. Linguistische Typologien der Kommunikation. Berlin, New York: de Gruyter, 275-295.

Bakhtin, Michail M. (1986): „The problem of speech genres“. In: Michail M. Bakhtin: Speech genres and other late essays. Hg. v. Caryl Emerson u. Michael Holquist. Austin: University of Texas Press, 60-102.

Bauman, Richard/Briggs, Charles L. (1990): „Poetics and performance as critical perspectives on language and social life“. In: Annual Review of Anthropology 19, 59-88.

Beaugrande, Robert-Alain de/Dressler, Wolfgang (1981): Einführung in die Textlinguistik. Tübingen: Niemeyer.

Birkner, Karin (2001): Bewerbungsgespräche mit Ost- und Westdeutschen. Eine kommunikative Gattung in Zeiten gesellschaftlichen Wandels Tübingen: Niemeyer.

Briggs, Charles L./Bauman, Richard (1992): „Genre, intertextuality, and social power“. In: Journal of Linguistic Anthropology 2(2), 131-172.

38 Ein ähnliches Phänomen wären etwa die mit dem Suffix -gate bezeichneten Skandale bzw. die durch diese Skandale ausgelösten öffentlichen Diskussionen (vgl. hierzu Stefanowitsch 2014). Diese gab es freilich schon vor der Etablierung des Internet als alläglichem Informationsund Kommunikationsmedium, doch erst seit wenigen Jahren und verstärkt durch die Möglichkeiten des Web 2.0, insbesondere das Hashtagging, werden verschiedenste Skandale gleichermaßen als Dönergate, Handygate, Dirndlgate uws. bezeichnet und zueinander in intertextuelle Bezüge gesetzt. 
Burger, Harald/Luginbühl, Martin $\left({ }^{4} 2014\right):$ Mediensprache. Eine Einführung in Sprache und Kommunikationsformen der Massenmedien. Berlin, Boston: de Gruyter.

Dammann, Günter (2000): „Textsorten und literarische Gattungen“. In: Klaus Brinker/Gerd Antos/Wolfgang Heinemann/Sven F. Sager (Hg.): Text- und Gesprächslinguistik. Ein internationales Handbuch zeitgenössischer Forschung. Bd. 1. Berlin, New York: de Gruyter, 546561.

Devitt, Amy J. (2004): Writing genres. Carbondale: Southern Illinois University Press.

Dürscheid, Christa (2005): „Medien, Kommunikationsformen, kommunikative Gattungen“. In: Linguistik online 22(1), 3-16.

Fiehler, Reinhard (1993): „Grenzfälle des Argumentierens. ,Emotionalität statt Argumentation“ oder ,emotionales Argumentieren““. In: Barbara Sandig/Ulrich Püschel (Hg.): Stilistik. Bd. 3: Argumentationsstile. Hildesheim, New York: Olms, 149-174.

Fiehler, Reinhard (2009): „Emotionale Kommunikation“. In: Ulla Fix/Andreas Gardt/Joachim Knape (Hg.): Rhetorik und Stilistik. Ein internationales Handbuch zeitgenössischer Forschung. Berlin, New York: de Gruyter, 757-772.

Fix, Ulla (2008): „Text- und Textlinguistik“. In: Nina Janich (Hg.): Textlinguistik. 15 Einführungen. Tübingen: Narr, 15-34.

Fix, Ulla (2014): „Aktuelle Tendenzen des Textsortenwandels - Thesenpapier“. In: Stefan Hauser/Ulla Kleinberger/Kersten Sven Roth (Hg.): Musterwandel - Sortenwandel. Aktuelle Tendenzen der diachronen Text(sorten)linguistik. Bern u. a.: Lang.

Fleischer, Wolfgang/Barz, Irmhild ( $\left.{ }^{2} 1995\right)$ : Wortbildung der deutschen Gegenwartssprache. Tübingen: Niemeyer.

Freadman, Anne (2002): „Uptake“. In: Richard M. Coe/Lorelei Lingard/Tatiana Teslenko (Hg.): The rhetoric and ideology of genre. Strategies for stability and change. Cresskill: Hampton, 39-53.

Fries, Norbert (2003): „de ira“. In: Linguistik online 13(1), 103-123.

Giltrow, Janet/Stein, Dieter (Hg.) (2009a): Genres in the Internet. Amsterdam, Philadelphia: Benjamins.

Giltrow, Janet/Stein, Dieter (2009b): „Genres in the internet. Innovation, evolution, and genre theory“. In: Janet Giltrow/Dieter Stein (Hg.): Genres in the internet. Amsterdam, Philadelphia: Benjamins, 1-25.

Gülich, Elisabeth (1986): „Textsorten in der Kommunikationspraxis“. In: Werner Kallmeyer (Hg.): Kommunikationstypologie. Handlungsmuster, Textsorten, Situationstypen. Düsseldorf: Schwann, 15-46.

Günthner, Susanne (1995): „Gattungen in der sozialen Praxis. Die Analyse ,kommunikativer Gattungen“ als Textsorten mündlicher Kommunikation“. In: Deutsche Sprache 23, 193-218.

Günthner, Susanne (2002): „Stimmenvielfalt im Diskurs. Formen der Stilisierung und Ästhetisierung in der Redewiedergabe“. In: Gesprächsforschung 3, 59-80.

Günthner, Susanne/Knoblauch, Hubert (1994): „,Forms are the food of faith'. Gattungen als Muster kommunikativen Handelns“. In: Kölner Zeitschrift für Soziologie und Sozialpsychologie 46(4), 693-723.

Habscheid, Stephan (2011a): „Das halbe Leben. Ordnungsprinzipien einer Linguistik der Kommunikation - Zur Einleitung in den Band“. In: Stephan Habscheid (Hg.): Textsorten, Handlungsmuster, Oberflächen. Linguistische Typologien der Kommunikation. Berlin, New York: de Gruyter, 3-29.

Habscheid, Stephan (Hg.) (2011b): Textsorten, Handlungsmuster, Oberflächen. Linguistische Typologien der Kommunikation. Berlin, New York: de Gruyter. 
Hagemann, Jörg (1997): Reflexiver Sprachgebrauch. Diktumscharakterisierung aus Gricescher Sicht. Opladen: Westdeutscher Verlag.

Hanks, William F. (1987): „Discourse genres in a theory of practice“. In: American Anthropologist 14(4), 668-692.

Hauser, Stefan/Kleinberger, Ulla/Roth, Kersten Sven (2014): „Textmuster und Textsorten im Wandel - Einführende Anmerkungen zu aktuellen Fragen der diachronen Textlinguistik“. In: Stefan Hauser/Ulla Kleinberger/Kersten Sven Roth (Hg.): Musterwandel - Sortenwandel. Aktuelle Tendenzen der diachronen Text(sorten)linguistik. Bern u. a.: Lang, 7-14.

Hauser, Stefan/Luginbühl, Martin (2015): „Hybridisierung und Ausdifferenzierung - Einführende begriffliche und theoretische Anmerkungen“. In: Stefan Hauser/Martin Luginbühl (Hg.): Hybridisierung und Ausdifferenzierung. Konstrastive Perspektiven linguistischer Medienanalyse. Bern u. a.: Lang, 7-30.

Heinemann, Wolfgang (2000): „Textsorte - Textmuster - Texttyp“. In: Klaus Brinker/Gerd Antos/ Wolfgang Heinemann/Sven F. Sager (Hg.): Text- und Gesprächslinguistik. Ein internationales Handbuch zeitgenössischer Forschung. Bd. 1. Berlin, New York: de Gruyter, 507-523.

Hempfer, Klaus W. (2010): „Zum begrifflichen Status der Gattungsbegriffe. Von ,Klassen“ zu ,Familienähnlichkeiten“ und ,Prototypen““. In: Zeitschrift für französische Sprache und Literatur 120(1), 14-32.

Heyd, Theresa (2009): „A model for describing ,new“ and ,old" properties of CMC genres. The case of digital folklore“. In: Janet Giltrow/Dieter Stein (Hg.): Genres in the internet. Amsterdam, Philadelphia: Benjamins, 239-262.

Hodapp, Volker (2000): „Ärger“. In: Jürgen H. Otto/Harald A. Euler/Heinz Mandl (Hg.): Emotionspsychologie. Ein Handbuch. Weinheim: Beltz, 199-208.

Holly, Werner (2002): „Fernsehspezifik von Präsentationsformen und Texttypen“. In: JoachimFelix Leonhard/Hans-Werner Ludwig/Dietrich Schwarze/Erich Straßner (Hg.): Medienwissenschaft. Ein Handbuch zur Entwicklung der Medien und Kommunikationsformen. Berlin, New York: de Gruyter, 2452-2464.

Holthuis, Susanne (1993): Intertextualität. Aspekte einer rezeptionsorientierten Konzeption. Tübingen: Stauffenburg.

Imo, Wolfgang (2007): Construction Grammar und Gesprochene-Sprache-Forschung. Konstruktionen mit zehn matrixsatzfähigen Verben im gesprochenen Deutsch. Tübingen: Niemeyer.

Imo, Wolfgang (2011): „,Hi! Danke fürs äddn.‘ Die Herausbildung einer neuen Floskel im Rahmen der social network website MySpace“. In: Martin Luginbühl/Daniel Perrin (Hg.): Muster und Variation. Medienlinguistische Perspektiven auf Textproduktion und Text. Bern u. a.: Lang, 125-155.

Jauß, Hans Robert (1977): „Theorie der Gattungen und Literatur des Mittelalters“. In: Hans Robert Jauß (Hg.): Alterität und Modernität der mittelalterlichen Literatur. Gesammelte Aufsätze 1956-1976. München: Fink, 327-358.

Keller, Eric (1981): „Gambits: Conversational strategy signals“. In: Florian Coulmas (Hg.): Conversational routine. Explorations in standardized communicative situations and prepatterned speech. New York: Mouton, 93-113.

Kövecses, Zoltan (1990): Emotion concepts. New Delhi, Berl, Heidelberg, London, Paris, Tokyo, Hong Kong: Springer.

Krause, Wolf-Dieter (2000): „Text, Textsorte, Textvergleich“. In: Kirsten Adamzik (Hg.): Textsorten. Reflexionen und Analysen. Tübingen: Stauffenburg, 45-76. 
Luginbühl, Martin (2014a): „Genre profiles and genre change“. In: Jannis Androutsopoulos (Hg.): Mediatization and sociolinguistic change. Berlin, Boston: de Gruyter, 305-330.

Luginbühl, Martin, (2014b): Medienkutur und Medienlinguistik. Komparative Textsortengeschichte(n) der amerikanischen „CBS Evening News“ und der Schweizer „Tagesschau“. Bern u. a.: Lang.

Luginbühl, Martin/Perrin, Daniel (2011): „,das, was wir in der Tagesschau den Rausschmeißer nennen': Altro- und Ethnokategorisierung von Textsorten im Handlungsfeld journalistischer Fernsehnachrichten“. In: Stephan Habscheid (Hg.): Textsorten, Handlungsmuster, Oberflächen. Linguistische Typologien der Kommunikation. Berlin, New York: de Gruyter, 577-596.

Marx, Konstanze/Weidacher, Georg (2014): Internetlinguistik. Ein Lehr- und Arbeitsbuch. Tübingen: Narr.

Meier, Georg Friedrich (1757): Versuch einer allgemeinen Auslegungskunst. Halle: Hemmerde.

Meier, Stefan (2008): (Bild-)Diskurs im Netz. Konzept und Methode für eine semiotische Diskursanalyse im World Wide Web. Köln: Herbert von Halem Verlag.

Meyer, Wolfgang (Hg.) (1913): Die Briefe Friedrich Ludwig Jahns. Leipzig: Eberhardt.

Miller, Carolyn R. (1984): „Genre as Social Action“. In: Quarterly Journal of Speech 70, 151-167.

Neumann, Uwe (1998): „Invektive“. In: Gert Ueding (Hg.): Historisches Wörterbuch der Rhetorik. $B d$. 4. Tübingen: Niemeyer, 549-561.

Niehüser, Wolfgang (1987): Redecharakterisierende Adverbiale. Göppingen: Kümmerle.

Pichl, Robert (1998): „Iracundia“. In: Gert Ueding (Hg.): Historisches Wörterbuch der Rhetorik. Tübingen: Niemeyer, 597-599.

Reisigl, Martin (2014): „Gattung“. In: Daniel Wrana/Alexander Ziem/Martin Reisigl/Martin Nonhoff/Johannes Angermuller (Hg.): DiskursNetz. Wörterbuch der interdisziplinären Diskursforschung. Berlin: Suhrkamp, 158.

Schwarz-Friesel, Monika ( $\left.{ }^{2} 2013\right):$ Sprache und Emotion. Tübingen, Basel: Francke.

Sendlmeier, Walter F. (2012): „Die psychologische Wirkung von Stimme und Sprechweise Geschlecht, Alter, Persönlichkeit, Emotion und audiovisuelle Interaktion“. In: Oksana Bulgakowa (Hg.): Resonanz-Räume - Die Stimme und die Medien. Berlin: Bertz + Fischer, 99-116.

Spiegel, Carmen (2011): Streit. Eine linguistische Untersuchung verbaler Interaktionen in alltäglichen Zusammenhängen. Mannheim: Verlag für Gesprächsforschung.

Spitzmüller, Jürgen (2013): Graphische Variation als soziale Praxis. Eine soziolinguistische Theorie skripturaler ,Sichtbarkeit‘. Berlin, Boston: de Gruyter.

Stefanowitsch, Anatol (2014): „And the winner is: -gate“. In: Sprachlog. http://www.sprachlog. de/2014/01/28/and-the-winner-is-gate/ [08.04.2015].

Stein, Stephan (1995): Formelhafte Sprache. Untersuchungen zu ihren pragmatischen und kognitiven Funktionen im gegenwärtigen Deutsch. Frankfurt a. M. u. a.: Lang.

Swales, John M. (1992): Genre analysis. English in academic and research settings. Cambridge: Cambridge University Press.

Tienken, Susanne (2015): „Neue Medien, neue Formen? Hybridisierung als Aspekt sozialen Wandels.“ In: Stefan Hauser/Martin Luginbühl (Hg.): Hybridisierung und Ausdifferenzierung. Konstrastive Perspektiven linguistischer Medienanalyse. Bern u. a.: Lang, 31-56.

Ulmer, Bernd/Bergmann, Jörg (1993): „Medienrekonstruktionen als kommunikative Gattungen?“. In: Werner Holly/Ulrich Püschel (Hg.): Medienrezeption als Aneignung. Methoden und Perspektiven qualitativer Medienforschung. Opladen: Westdeutscher Verlag, 81-102.

Voßkamp, Wilhelm (1997): „Gattungsgeschichte“. In: Klaus Weimar (Hg.): Reallexikon der deutschen Literaturwissenschaft. Berlin, New York: de Gruyter, 655-658. 
Wierzbicka, Anna (1999): Emotions across languages and cultures. Diversity and universals. Cambridge: Cambridge University Press.

Wittgenstein, Ludwig (1984): Werkausgabe Bd. 1: Tractatus Logico-philosophicus. Tagebücher 1914-1916. Philosophische Untersuchungen. Frankfurt a. M.: Suhrkamp.

Zifonun, Gisela/Hoffmann, Ludger/Strecker, Bruno (1997): Grammatik der deutschen Sprache. 3 Bände. Berlin, New York: de Gruyter.

Zymner, Rüdiger (2010): „Biopoetische/Kognitionswissenschaftliche Gattungstheorie“. In: Rüdiger Zymner (Hg.): Handbuch Gattungstheorie. Stuttgart: Metzler, 162-164. 